\title{
Concordance between Oropharyngeal Flora and Peristomal Wound Culture Bacteria Following Percutaneous Endoscopic Gastrostomy: A Prospective Cohort Study
}

\author{
Da Hwi Jung, M.D. ${ }^{1}$, Dong Hoon Baek, Ph.D. ${ }^{2}$, Jin A Yoon, Ph.D. ${ }^{1}$, Sang Hun Kim, M.D. ${ }^{1}$, \\ Byeong Ju Lee, M.D. ${ }^{1}$, Mi Kyung Cho, M.D. ${ }^{1}$, Yong Beom Shin, Ph.D. ${ }^{1}$ \\ ${ }^{1}$ Department of Rehabilitation Medicine, Pusan National University Hospital, Pusan National University School of \\ Medicine and Biomedical Research Institute, Busan, ${ }^{2}$ Department of Internal Medicine, Pusan National University \\ Hospital, Pusan National University School of Medicine and Biomedical Research Institute, Busan, Korea
}

\begin{abstract}
Objective: To examine the concordance between the oropharyngeal flora and peristomal wound culture bacteria following percutaneous endoscopic gastrostomy (PEG) placement.

Methods: Cultures of oropharyngeal flora before PEG placement and peristomal wound cultures after PEG placement were examined prospectively in patients who underwent PEG between June 1, 2017, and June 1, 2019, at a local rehabilitation center. Microorganisms in the oropharyngeal flora and peristomal wound culture were identified, and the relationship between them was examined. The risk factors that may increase the PEG infection rate were also examined.

Results: The predominant bacterium identified in the oropharyngeal flora and peristomal wounds in patients who required PEG was Pseudomonas aeruginosa. The concordance between the oropharyngeal flora and peristomal wound culture bacteria in patients with PEG infections was confirmed. Different oropharyngeal flora was observed in patients with a prolonged hospital stay compared to that of healthy adults. The concordance between the oropharyngeal flora and peristomal wounds was also identified with a high prevalence of gram-negative bacilli. There was no potential risk factor, such as comorbidities or length of hospitalization of patients associated with PEG infections. Conclusion: Because the oropharyngeal flora showed concordance with the bacteria found in the PEG stoma site, management of the oropharyngeal flora before PEG insertion should be considered for patients with dysphagia. (JKDS 2021;11:35-42)
\end{abstract}

Keywords: Enteral nutrition, Gastrostomy, Oropharynx, Oral hygiene, Surgical wound infection

Received: April 29 2020, Revised: May 6 2020,

Accepted: June 122020

Corresponding author: Yong Beom Shin, Department of Rehabilitation Medicine, Pusan National University Hospital, Pusan National University School of Medicine and Biomedical Research Institute, 179 Gudeok-ro, Seo-gu, Busan 49241, Korea Tel: +82-51-240-7485, Fax: +82-51-247-7485

E-mail: yi0314@gmail.com
Copyrights (c) The Korean Dysphagia Society, 2021. 


\section{INTRODUCTION}

Causes of swallowing disorders include stroke, traumatic brain injury, head and neck cancer, motor neuron disease, and aging ${ }^{1}$. Prolonged difficulty with swallowing may require patients to receive enteral or parenteral nutrition. Enteral nutrition has several advantages over parenteral nutrition, such as fewer infectious complications and reduced length of stay ${ }^{2}$; therefore, enteral nutrition should be introduced in these patients. However, long-term use of a nasogastric tube may result in complications, such as gastro-esophageal reflux and aspiration pneumonia since the lower esophageal sphincter remains open because of the tube placement ${ }^{3}$. In order to avoid the complications associated with long-term use of a nasogastric tube, percutaneous endoscopic gastrostomy (PEG) is used for long-term nutritional support in those who have swallowing difficulties.

Despite its advantages, PEG tube placement is associated with several complications, such as focal wound infection due to stoma site leakage or peritonitis. Pneumoperitoneum, aspiration pneumonia, buried bumper syndrome and necrotizing fasciitis are also known as major complication of PEG tube insertion. Of these, peristomal wound infection is the most common complication with various prevalence between 5 and $65 \%$ compared to $18 \%$ of aspiration pneumonia ${ }^{4}$. There are several methods of PEG insertion: push type, introducer type and pull type. The most commonly used type is the pull technique ${ }^{5}$. In this technique, a tube is placed in the patient's stomach via the mouth through the esophagus so that the guide wire reaches the stomach and the PEG catheter can be safely inserted through the abdominal wall. We hypothesized that the oropharyngeal flora causes PEG site infection through contamination of the tip of the PEG catheter during this procedure.

In this study, we aimed to analyze and evaluate the concordance between the cultures of the oropharyngeal flora and peristomal wound culture following PEG placement in patients with a prolonged hospital stay at a rehabilitation center. The findings may enable us to determine preventive measures to reduce the risk of PEG infection in patients suffering from impaired swallowing function and malnutrition.

\section{MATERIALS AND METHODS}

\section{Patients}

Patients who were admitted to a rehabilitation center and underwent PEG placement between June 1, 2017 and June 1, 2019 were recruited in this study. Patients with active systemic infections with clinical symptoms before PEG insertion were excluded. Written informed consent was obtained. This study was approved by the Pusan National University Hospital Institutional Review Board (IRB No. H-1909-004-082).

\section{Cultures and evaluations}

A single culture swab obtained each from the base of the tongue and palatopharyngeal arch 1 day before PEG placement was used to determine the baseline oropharyngeal flora. A culture swab was also performed at the stoma site in those who underwent tracheostomy. We administered $2 \mathrm{~g}$ of ceftriaxone intravenously before PEG insertion. A peristomal culture was obtained on the day after the procedure. After the procedure, thorough examinations were performed during dressing the PEG site for 1 week. An additional PEG culture was obtained 3-7 days after the procedure in patients with signs of local infection, such as peristomal erythema, induration, and purulent discharge. All cultures were obtained using the Levine's technique ${ }^{6}$. An additional peristomal culture was obtained in patients with diagnosed PEG infection. PEG infection was deemed present when there were signs of systemic infection, such as fever, leukocytosis, and elevated C-reactive protein, while excluding possibility of urinary tract infection and aspiration pneumonia. These patients were treated with appropriate systemic antibiotics. Concordances between the oropharyngeal flora, peristomal wound culture bacteria on postoperative day (POD)-1, POD3-7, and on the day of diagnosis of PEG infection were examined. 
Sputum cultures obtained from patients who had clinical signs of respiratory tract infections at 7 days before and after PEG placement were also examined to assess the concordance between sputum cultures and PEG infection.

\section{Data collection}

The patients' previous medical history, such as diabetes mellitus and hypertension, was examined to assess for factors contributing to PEG infections. Duration of the disease and length of hospitalization were also included. Respiratory function was assessed on the basis of the status of tracheostomy and use of portable ventilators. The Penetration Aspiration Scale (PAS) obtained from videofluoroscopic swallowing study of each patient was used to assess swallowing function. Additionally, we examined the phase of swallowing (oral preparatory, oral propulsive, laryngeal, and esophageal) that was interrupted. The functional status was evaluated using the Eastern Cooperative Oncology Group (ECOG) performance status. The Korean version of the Modified Barthel Index (K-MBI) was used to evaluate the patients with brain lesions, and the Amyotrophic Lateral Sclerosis Functional Rating Scale-Revised (ALS-FRS-R) score was used to assess the patients with neuromuscular diseases. Serum albumin level was used as an indicator of nutritional status since body weight could not be accurately estimated in patients due to their decreased mobility.

\section{Statistical analysis}

The kappa coefficient $(\kappa)$ between the oropharyngeal or pharyngeal flora and peristomal wound culture bacteria was estimated to examine the concordance between the $\mathrm{two}^{7}$. The percentages of identical microorganisms found on both oropharyngeal and peristomal wound cultures were examined in all patients. Fisher's exact test and T-test were used to assess the risk of PEG infection between groups of patients with and without PEG infections. Differences were considered significant when the P-value was less than 0.05. All data were analyzed using SPSS version 22
(IBM Corp., Armonk, NY, USA).

\section{RESULTS}

\section{Patient demographics}

A total of 52 patients in the local rehabilitation center were enrolled among total 109 patients in the hospital where the study was conducted in the study period, with a mean age of $60.9 \pm 14.6$ years. There were 30 males with a mean duration of disease of $268.1 \pm 346.2$ days and a mean duration of hospitalization of $44.2 \pm 42.7$ days. Table 1 summarized the overall demographic data these patients.

Table 1. Demographic data of the patients.

\begin{tabular}{lc} 
Sex & $30(57.7 \%): 22(42.3 \%)$ \\
Male:Female & $60.9 \pm 14.6$ \\
Mean Age & $40(76.9 \%)$ \\
Diagnosis & $8(15.4 \%)$ \\
Brain lesion & $1(1.9 \%)$ \\
MND & $3(5.8 \%)$ \\
Head \& Neck & $9(17.3 \%)$ \\
Pneumonia & $6(11.5 \%)$ \\
Past history & $1(1.9 \%)$ \\
DM & $5(9.6 \%)$ \\
HT & $7(13.5 \%)$ \\
Parkinsonism & $23(44.2 \%)$ \\
Old CVA & $268.1 \pm 346.2$ \\
Others & $44.2 \pm 42.7$ \\
None & $5.23 \pm 3.29$ \\
Duration of disease & \\
Length of Hospitalization & $47(90.4 \%): 5(9.6 \%)$ \\
PAS score & \\
Tracheostomy state & $12(23.1 \%): 39(75.0 \%)$ \\
Yes:No & \\
Home vent apply & $23(44.2 \%): 29(55.8 \%)$ \\
Yes:No & $3.9 \pm 0.4$ \\
Sputum culture & $5.0 \pm 12.4$ \\
Positive:Negative & $6.4 \pm 10.3$ \\
Functional status & $3.4 \pm 0.4$ \\
ECOG Performance Status & \\
K-MBI & \\
ALS-FRS-R & \\
Serum Albumin (g/dL) & \\
\hline
\end{tabular}

MND: motor neuron disease, DM: diabetes mellitus, HT: hypertension, CVA: cerebrovascular accident, PAS: Penetration-Aspiration Scale, ECOG: Eastern Cooperative Oncology Group Performance Status, K-MBI: Korean Version of Modified Barthel Index, ASL-FRS-R: Amyotrophic Lateral Sclerosis Functional Rating Scale-Revised, g: gram, dL: deciliter. 


\section{Microorganisms in the oropharynx and peristomal wound site}

The microorganisms that were identified in the oropharynx and peristomal wound site are illustrated in Fig. 1 and Table 2. The most commonly identified

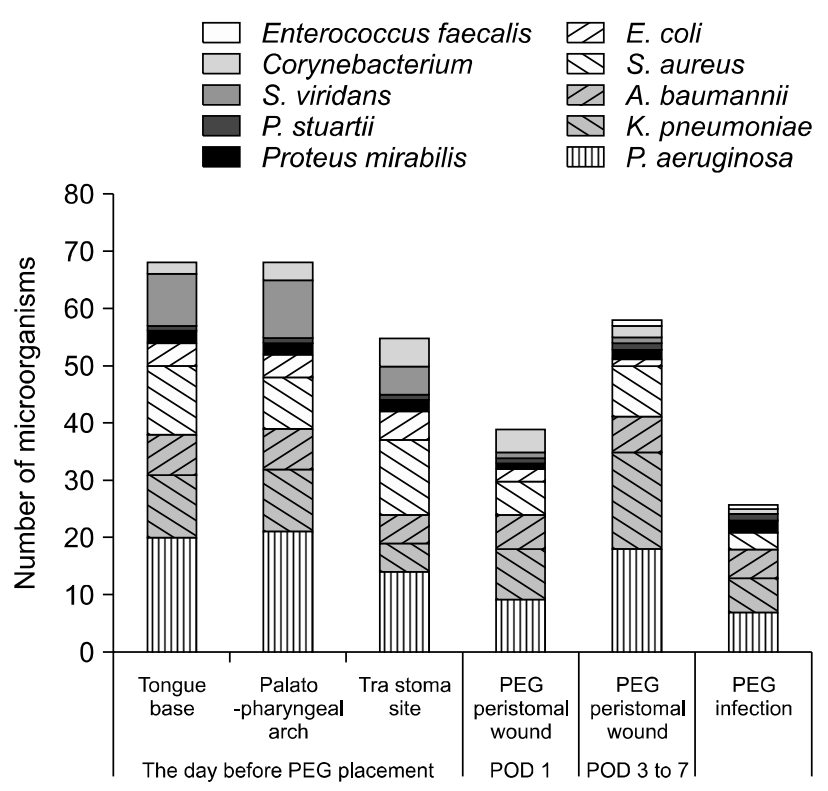

Fig. 1. Microorganisms identified in the oropharynx and peristomal wound before and after PEG placement.

PEG: percutaneous endoscopic gastrostomy, P. aeruginosa: Pseudomonas aeruginosa, $K$. pneumoniae: Klebsiella pnuemoniae, A. baumanii. Acinetobacter baumanii, S. aureus: Staphylococcus aureus, P. mirabilis: Proteus mirabilis, P. stuartii. Providencia stuartii. gram-negative bacteria at the base of the tongue, palatopharyngeal arch, and PEG placement site were Pseudomonas aeruginosa, Klebsiella pneumoniae, Acinetobacter baumannii, and Escherichia coli, while Staphylococcus aureus, Streptococcus viridans, and Corynebacterium species were the most commonly identified gram-positive bacteria.

PEG infection was present in 16/52 patients (30.8\%) after an average of 8 days (mean \pm SD: $8.13 \pm 6.82$ ). An additional peristomal wound culture was performed when PEG infection was diagnosed, and Fig. 2 illustrates the prevalence of microorganisms that were identified at the peristomal wound site when PEG infection was present. The most common organism found throughout the oropharynx, peristomal wound, and sputum cultures was $P$. aeruginosa, a gramnegative bacterium. All $S$. aureus were Methicillinresistant $S$. aureus (MRSA).

\section{Concordance between oropharyngeal flora and peristomal wound cultures}

Among 16 patients with PEG infection, 12 patients showed same bacterium identified at both oropharynx and peristomal PEG site while 3 patients showed different bacterium at both sites and one patient showed no growth of bacteria at PEG site. Among 12 patients who showed same bacterial identification at both sites, a single bacterium was

Table 2. Microorganisms identified in the oropharynx flora and the peristomal wound.

\begin{tabular}{|c|c|c|c|c|c|c|}
\hline & \multicolumn{3}{|c|}{ The day before PEG placement } & \multirow{2}{*}{$\frac{\text { POD } 1}{\text { PEG peristomal wound }}$} & \multirow{2}{*}{$\begin{array}{c}\text { POD } 3 \text { to } 7 \\
\text { PEG peristomal wound }\end{array}$} & \multirow{2}{*}{$\begin{array}{c}\text { PEG } \\
\text { infection }\end{array}$} \\
\hline & Tongue base & Palato-pharyngeal arch & Tra stoma site & & & \\
\hline P. aeruginosa & 20 & 21 & 14 & 9 & 18 & 7 \\
\hline K. pneumoniae & 11 & 11 & 5 & 9 & 17 & 6 \\
\hline A. baumannii & 7 & 7 & 5 & 6 & 6 & 5 \\
\hline S. aureus & 12 & 9 & 13 & 6 & 9 & 3 \\
\hline E. coli & 4 & 4 & 5 & 2 & 1 & 0 \\
\hline P. mirabilis & 2 & 2 & 2 & 1 & 2 & 2 \\
\hline P. stuartii & 1 & 1 & 1 & 1 & 1 & 1 \\
\hline S. viridans & 9 & 10 & 5 & 1 & 1 & 0 \\
\hline Corynebacterium & 2 & 3 & 5 & 4 & 2 & 1 \\
\hline E. faecalis & 0 & 0 & 0 & 0 & 1 & 1 \\
\hline
\end{tabular}

P. aeruginosa: Pseudomonas aeruginosa, S. aureus: Staphylococcus aureus, A. baumanii: Acinetobacter baumanii, K. pneumoniae: Klebsiella pnuemoniae, P. mirabilis: Proteus mirabilis, P. stuartii. Providencia stuartii, E. faecalis: Enterococcus faecalis, POD: postoperative day, tra: tracheostomy, PEG: percutaneous endoscopic gastrostomy. 


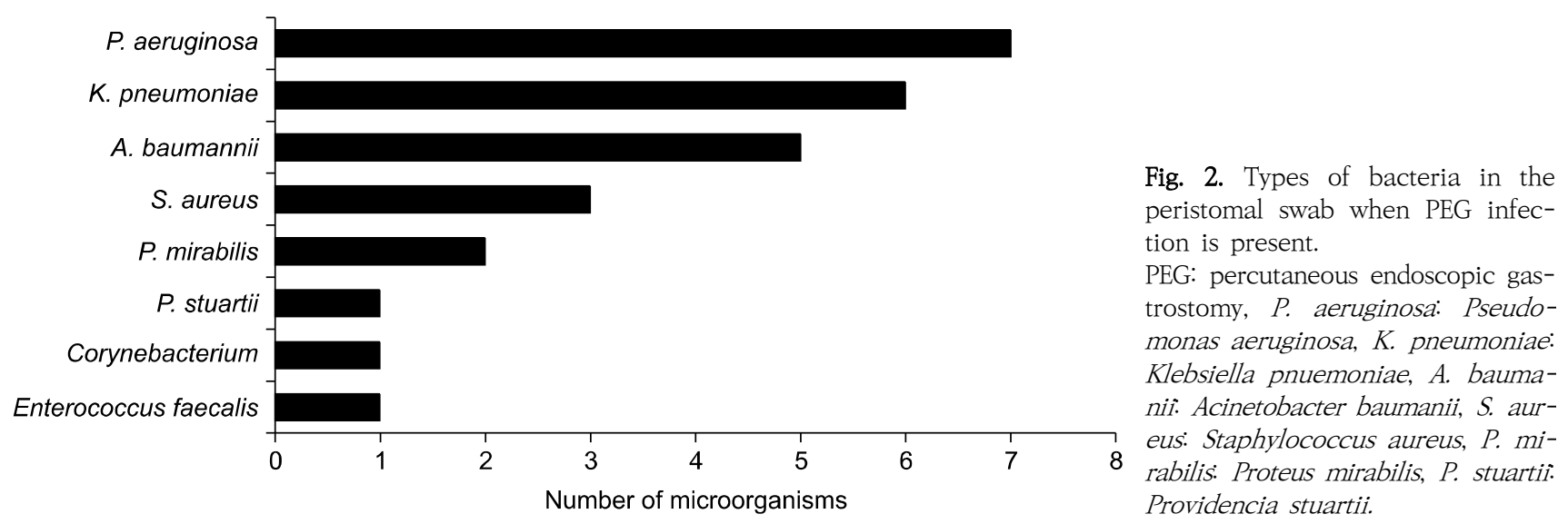

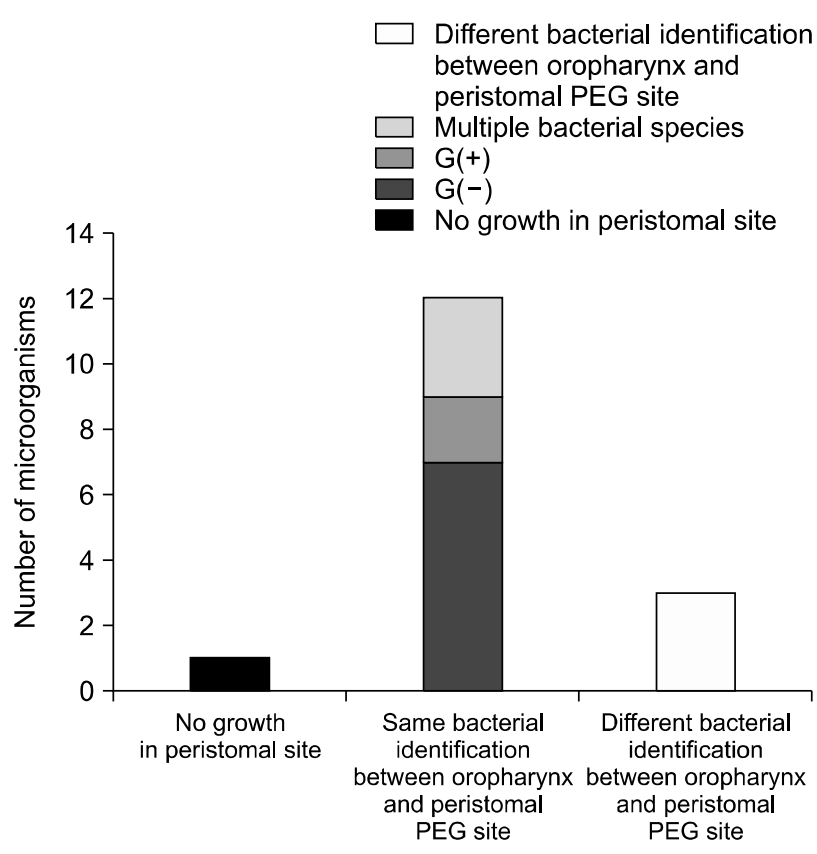

Fig. 3. The number of patients with different growths at the peristomal wound after PEG.

PEG: percutaneous endoscopic gastrostomy, G (-): Gram negative bacteria ( $P$. aeruginosa, $K$. pneumoniae, A. baumannii, Proteus mirabilis, Providencia stuartii, Corynebacterium species, E. faecalis), G (+): Gram positive bacteria ( $S$. aureus).

identified in 9 patients, and multiple bacteria-both gram-negative bacilli and gram-positive cocci-were identified in 3 patients. In 9 patients who were infected by a single bacterium, gram-negative bacteria were identified in 7 patients and the grampositive bacteria, $\mathcal{S}$. aureus was identified in the remaining 2.(Fig. 3) In 16 patients with PEG infections, concordance between the oropharyngeal
Table 3. Concordance between oropharyngeal flora and PEG site cultures.

\begin{tabular}{lccc}
\hline \multicolumn{1}{c}{ Site } & POD 1 & POD 3-7 & PEG infection \\
\hline Tongue base & 0.656 & 0.635 & 0.717 \\
Palatopharyngeal Arch & 0.664 & 0.684 & 0.736 \\
Tra stoma site & 0.643 & 0.624 & 0.730 \\
\hline
\end{tabular}

POD: postoperative day, PEG: percutaneous endoscopic gastrostomy, tra: tracheostomy.

flora and peristomal wound culture bacteria was observed in 14 (87.5\%) patients with substantial agreement ( $\kappa$ mean \pm SD:0.68 \pm 0.04 ). (Table 3) Table 4 summarizes the concordance of each microorganism between the oropharynx and peristomal wound when PEG infection was present. Percentage of each bacterium found in both oropharynx and peristomal wound were over $50 \%$ in three most common bacterium and $100 \%$ in 4 bacterium.(Table $4-a)$ Moreover, Among 14 patients who showed concordance between oropharyngeal flora and peristomal wound culture, 9 patients out of 14 (64.29\%) showed identical flora between oropharynx and peristomal wound. (Table 4-b)

Additionally, 7 patients out of 23 patients with positive sputum cultures demonstrated concordance between the oropharyngeal and peristomal wound cultures. Only 2/7 of these patients demonstrated no concordance of microorganisms between the peristomal wound and sputum cultures. 
Table 4. Concordance between oropharynx and peristomal wound when PEG infection is present.

\begin{tabular}{|c|c|c|c|c|c|c|c|c|c|}
\hline \multicolumn{5}{|c|}{$\begin{array}{l}\text { 4-a. Concordance of each bacteria between oropharynx and } \\
\text { peristomal wound. }\end{array}$} & \multicolumn{5}{|c|}{$\begin{array}{l}\text { 4-b. Percentage of number of identical microorganisms with } \\
\text { concordance between oropharynx and PEG infection site. }\end{array}$} \\
\hline & Oropharynx & $\begin{array}{l}\text { Peristomal } \\
\text { wound }\end{array}$ & Concordance & $\begin{array}{c}\% \text { of } \\
\text { concordance }\end{array}$ & & $\begin{array}{l}\text { Number of } \\
\text { Microrganisms }\end{array}$ & $\begin{array}{c}\text { Number of } \\
\text { Identical } \\
\text { Microorganisms }\end{array}$ & $\begin{array}{c}\text { Number } \\
\text { of } \\
\text { patients }\end{array}$ & $\begin{array}{c}\% \text { of } \\
\text { concordance }\end{array}$ \\
\hline P. aeruginosa & 6 & 7 & 4 & $57.14 \%$ & Concordance & 14 & 1 out of 1 & 5 & $100.00 \%$ \\
\hline$K$ pneumoniae & 3 & 6 & 4 & $66.67 \%$ & & & 2 out of 2 & 4 & $100.00 \%$ \\
\hline A. baumannii & 5 & 5 & 4 & $80.00 \%$ & & & 1 out of 3 & 1 & $66.67 \%$ \\
\hline S. aureus & 3 & 3 & 3 & $100.00 \%$ & & & 1 out of 2 & 4 & $50.00 \%$ \\
\hline P. mirabilis & 2 & 2 & 2 & $100.00 \%$ & Disconcordance & 2 & & & \\
\hline P. stuartii & 1 & 1 & 1 & $100.00 \%$ & Total & 16 & & & \\
\hline Corynebacterium & 1 & 1 & 1 & $100.00 \%$ & & & & & \\
\hline E. faecalis & 0 & 1 & 0 & $0.00 \%$ & & & & & \\
\hline
\end{tabular}

PEG: percutaneous endoscopic gastrostomy, P. aeruginosa: Pseudo- PEG: percutaneous endoscopic gastrostomy, \%: percentage. monas aeruginosa, $K$. pneumoniae: Klebsiella pnuemoniae, $A$. baumanir. Acinetobacter baumanii, S. aureus: Staphylococcus aureus, P. mirabilis: Proteus mirabilis, P. stuartii: Providencia stuartii, E. faecalis: Enterococcus faecalis.

Table 5. Risk factors associated with PEG infection.

\begin{tabular}{lccc}
\hline & \multicolumn{3}{c}{ Mean \pm SD } \\
\cline { 2 - 4 } & $\begin{array}{c}\text { PEG } \\
\text { infection } \\
(\mathrm{N}=16)\end{array}$ & $\begin{array}{c}\text { No PEG } \\
\text { infection } \\
(\mathrm{N}=36)\end{array}$ & P-value \\
\hline Age & $57.06 \pm 17.01$ & $64.61 \pm 12.91$ & 0.084 \\
Sex (Male:Female) & $8: 8$ & $22: 14$ & 0.548 \\
Diagnosis & - & - & 0.888 \\
Past history & - & - & 0.158 \\
Mentality & - & - & 0.276 \\
Tracheostomy state, \% (n) & 16 & 31 & 0.308 \\
Vent apply state, \% (n) & 4 & 8 & 0.730 \\
Pneumonia, \% (n) & 4 & 8 & 1.000 \\
Positive sputum culture, \% (n) & 8 & 15 & 0.763 \\
Duration of disease (day) & $114 \pm 78.78$ & $336.64 \pm 65.89$ & $0.031^{*}$ \\
Period of hospitalization (day) & $46.50 \pm 36.20$ & $43.11 \pm 45.69$ & 0.794 \\
PAS score & $2.31 \pm 3.42$ & $3.47 \pm 3.69$ & 0.290 \\
ECOG Functional Status & $4.00 \pm 0.00$ & $3.58 \pm 0.97$ & 0.093 \\
K-MBI & $0.88 \pm 3.50$ & $5.56 \pm 13.75$ & 0.188 \\
ALS-FRS-R & $0.25 \pm 1.00$ & $1.31 \pm 5.32$ & 0.437 \\
Serum albumin & $3.40 \pm 0.38$ & $3.44 \pm 0.38$ & 0.750 \\
\hline
\end{tabular}

PAS: Penetration-Aspiration Scale, ECOG: Eastern Cooperative Oncology Group Performance Status, K-MBI: Korean Version of Modified Barthel Index, ASL-FRS-R: Amyotrophic Lateral Sclerosis Functional Rating Scale-Revised, PEG: percutaneous endoscopic gastrostomy, SD: standard deviation.

*Statistically significant at a significance level of 0.05 .

\section{Risk factors associated with PEG infections}

No significant risk factor, except for duration of the disease, was associated with PEG infections. The disease duration was significantly longer, with a Pvalue of 0.031 , in those without PEG infections than that in those with PEG infections.(Table 5)

\section{DISCUSSION}

We found concordance between bacterial oropharyngeal flora and peristomal wound cultures in patients who underwent PEG placement in this study. The sputum cultures obtained from patients with upper airway infections also demonstrated concordance with the peristomal wound cultures. Unlike normal flora of the skin and mouth, gram-negative bacilli were the predominant ones identified in the oropharynx and peristomal sites in our patients. There was no significant risk factor associated with PEG infections.

The pull technique is the most frequently used method in PEG placement. There are several methods to reduce the risk of PEG infections during the procedure, such as cleaning the abdominal wall with povidone spray and suctioning the oral cavity to reduce aspiration of saliva ${ }^{8}$. Antibiotics, a single dose of first or third generation cephalosporin, are recommended to be applied prior to the procedure ${ }^{8}$, however, the use of prophylactic antibiotics is still con- 
troversial. Additionally, several studies have reported nosocomial infections and that therefore prophylactic antibiotics should cover MRSA ${ }^{9-11}$.

There are several studies have reported concordance between the oropharyngeal bacteria and PEG infections. Two of them suggested that MRSA causes PEG infections because the bacteria are colonized in the oropharynx and transferred by the pull technique during PEG placement ${ }^{8-10}$. Our findings also demonstrated that $S$. aureus is a common microorganism found in the oropharynx and at peristomal wound sites. In another study that investigated the correlation between oropharyngeal microorganisms and PEG infections, a significant increase in the PEG infection rate was observed in the presence of nasopharyngeal colonization ${ }^{12}$. As many as $28.1 \%$ to $36 \%^{10,12}$ of PEG infection were shown in those studies ${ }^{8-10,12}$, similar to $30.8 \%$ of PEG infection rate of this study.

Causative microorganisms for PEG infection are present during the pull technique since normal flora of the skin and oropharyngeal flora are present in the layers that the catheter passes through. The predominant bacteria from the normal skin are usually gram-positive bacteria, such as $S$. epidermidis and $S$. aureus $^{13}$. However, the predominant species identified at the PEG stoma site in this study were gramnegative. Moreover, in the oropharyngeal flora of healthy adults, Staphylococci are the predominant organisms. However, the oropharyngeal flora of inpatients with prolonged hospital stay demonstrate changes from the normal oropharyngeal flora ${ }^{14}$. Our findings also demonstrated that the predominant microorganisms in the oropharynx of enrolled patients were gram-negative bacteria,(Fig. 1) unlike in the normal oropharyngeal flora. The most common organism found throughout the oropharynx, peristomal wound, and sputum cultures was $P$. aeruginosa. Fig. 2 illustrates the prevalence of microorganisms that were identified at the peristomal wound site when PEG infection was present, showing predominancy of gram-negative bacteria. In addition to this, the frequencies of gram-negative microorganisms found in PEG cultures on POD-1, POD-3-7, and when
PEG infection was diagnosed, were similar.(Table 1) Furthermore, concordance between the bacteria of the oropharynx and peristomal wound was observed. (Table 3) With similar dominancy of species found in the PEG stoma site and oropharyngeal flora with concordance, PEG infection are related with oropharyngeal flora.

The risk factors for PEG insertion have been widely studied to reduce the risk of PEG infections, and history of diabetes mellitus or cancer, current infection, and decreased functional status are considered as some of the possible risk factors ${ }^{15-17}$. However, except for the duration of the disease, no significant risk factor for PEG infection was identified in this study. Patients with shorter disease duration was shown to have increased rates of PEG infection, as patients with longer periods of illness are generally admitted after outpatient treatment, and hospital stays are shorter than those recently diagnosed. Since that was not our main goal, the relationship between hospital stay, disease duration and PEG infection rate altogether was not fully discussed. Nevertheless, risk factors associated with PEG infection should be discussed in further studies.

\section{Limitations}

This study has a few limitations. First, there was inconsistency in the method of oral hygiene care because of different swallowing functions between the patients. Patients who did not undergo thorough oral care would have high risk of PEG infection. Chlorhexidine gluconate solution is known to be a good antiplaque agent and also serves as antimicrobial agent with broad spectrum, including gram negative and positive bacteria as well as yeast ${ }^{18}$. However, soley cleansing oral with soaked gauze with chlorhexidine solution is not enough to eliminate plaque of tongue and teeth. Therefore, several tool such as suctioning brush and interdental tooth brush can be used for additional oral hygiene care. In further study, PEG infection rate after various oral hygiene method can be discussed to find proper oral hygiene care. Secondly, since ceftriaxone, a third- 
generation cephalosporin used in our study, is inactive against MRSA and only has moderate activity against Pseudomonas and Acinetobacter species completely ${ }^{19,20}$. Since ceftriaxone was administered to every patients enrolled in this study before PEG insertion, it would have an effect on culture of PEG stoma site. Proper types of prophylactic antibiotics can be discussed in further study.

\section{CONCLUSION}

We found the concordance between oropharyngeal flora and PEG stoma infection in this study. There are many preventive methods to reduce PEG infection after the procedure so far. As concordance between oropharynx and PEG stoma site was found in this study, further study designed for management of oropharyngeal flora before PEG insertion could be considered for patients with dysphagia.

\section{ACKNOWLEDGEMENTS}

This work was supported by a 2-year Research Grant of Pusan National University. We would like to thank Dr. Lee for helping us organize the statistical data.

\section{REFERENCES}

1. Rahnemai-Azar AA, Rahnemaiazar AA, Naghshizadian R, Kurtz A, Farkas DT. Percutaneous endoscopic gastrostomy: indications, technique, complications and management. World J Gastroenterol 2014;20:7739-51.

2. Seres DS, Valcarcel M, Guillaume A. Advantages of enteral nutrition over parenteral nutrition. Therap Adv Gastroenterol 2013;6:157-67.

3. Prabhakaran S, Doraiswamy V, Nagaraja V, Cipolla J, Ofurum U, Evans D, et al. Nasoenteric tube complications. Scand J Surg 2012;101:147-55.

4. Rajput A, Waseem SA, Khanzada TW, Shaikh GM, Channa GA. Mortality in necrotizing fasciitis. J Ayub Med Coll Abbottabad 2008;20:96-8.

5. Lim YJ, Yang CH. Technique, Management and complications of percutaneous endoscopic gastrostomy. Korean J Gastrointest Endosc 2009;39(3):119-24.
6. Smith ME, Robinowtiz N, Chaulk P, Johnson K. Comparison of chronic wound culture techniques: swab versus curetted tissue for microbial recovery. $\mathrm{Br} \mathrm{J}$ Community Nurs. 2014;19(90):S22-S26.

7. Viera AJ, Garrett JM. Understanding interobserver agreement: the Kappa statistic. Family Med 2005;37:360-63.

8. Lynch CR, Fang JC. Prevention and management of complications of percutaneous endoscopic gastrostomy (PEG) tubes. Pract gastroenterol 2004;28:66-76.

9. Chaudhary KA, Smith OJ, Cuddy PG, Clarkston WK. PEG site infections: the emergence of methicillin resistant Staphylococcus aureus as a major pathogen. Am J Gastroenterol 2002;97:1713.

10. Horiuchi A, Nakayama Y, Kajiyama M, Fujii H, Tanaka $\mathrm{N}$. Nasopharyngeal decolonization of methicillin-resistant Staphylococcus aureus can reduce PEG peristomal wound infection. Am J Gastroenterol 2006;101:274.

11. Hull M, Beane A, Bowen J, Settle C. Methicillin-resistant Staphylococcus aureus infection of percutaneous endoscopic gastrostomy sites. Aliment Pharmacol Ther 2001;15: 1883-8.

12. Faias S, Cravo M, Claro I, Lage P, Nobre-Leitão C. High rate of percutaneous endoscopic gastrostomy site infections due to oropharyngeal colonization. Dig Dis Sci 2006;51:2384-8.

13. Cogen A, Nizet V, Gallo R. Skin microbiota: a source of disease or defence? Br J Dermatol 2008;158:442-55.

14. Johanson WG, Pierce AK, Sanford JP. Changing pharyngeal bacterial flora of hospitalized patients: emergence of gram-negative bacilli. N Eng J Med 1969;281:1137-40.

15. Kroupa R, Jurankova J, Dastych M, Senkyrik M, Pavlik T, Prokesova J, et al. Different clinical utility of oropharyngeal bacterial screening prior to percutaneous endoscopic gastrostomy in oncological and neurological patients. Biomed Res Int 2014;2014.

16. You CR, Kim SW, Paik CN, Park JM, Cho YK, Lee IS, et al. A risk factor for wound infection after percutaneous endoscopic gastrostomy: decreased performance status. Korean J Gastrointest Endosc 2007;35:133-9.

17. Lee HH, Shim KN, Kwon JM, Jung SA, Yoo K. Complications of percutaneous endoscopic gastrostomy and risk factors of peristomal wound infection. Korean J Med 2004; 67:15-21.

18. Kim EK, Jang SH, Choi YH, Lee KS, Kim YJ, Kim SH, et al. Effect of an Oral Hygienic Care Program for Stroke Patients in the Intensive Care Unit. Yonsei Med J. 2014; 55(1):240-46.

19. Richards DM, Heel RC, Brogden RN, Speight TM, Avery GS. Ceftriaxone. A review of its antibacterial activity, pharmacological properties and therapeutic use. Drugs 1984;27(6):469-527.

20. Cleeland R, Squires E. Antimicrobial activity of ceftriaxone: a review. Am J Med. 1984;77(4C):3-11. 